\title{
Accuracy of a reverse dot blot hybridization assay for simultaneous detection of the resistance of four anti-tuberculosis drugs in Mycobacterium tuberculosis isolated from China
}

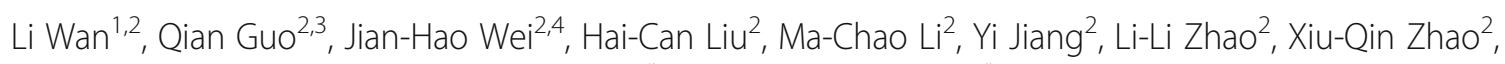
Zhi-Guang Liu², Kang-Lin Wan², Gui-Lian Li ${ }^{2 *}$ and Cha-Xiang Guan ${ }^{*}$

\begin{abstract}
Background: Drug resistant tuberculosis poses a great challenge for tuberculosis control worldwide. Timely determination of drug resistance and effective individual treatment are essential for blocking the transmission of drug resistant Mycobacterium tuberculosis. We aimed to establish and evaluate the accuracy of a reverse dot blot hybridization (RDBH) assay to simultaneously detect the resistance of four anti-tuberculosis drugs in M. tuberculosis isolated in China.

Methods: In this study, we applied a RDBH assay to simultaneously detect the resistance of rifampicin (RIF), isoniazid (INH), streptomycin (SM) and ethambutol (EMB) in 320 clinical M. tuberculosis isolates and compared the results to that from phenotypic drug susceptibility testing (DST) and sequencing. The RDBH assay was designed to test up to 42 samples at a time. Pearson's chi-square test was used to compute the statistical measures of the RDBH assay using the phenotypic DST or sequencing as the gold standard method, and Kappa identity test was used to determine the consistency between the RDBH assay and the phenotypic DST or sequencing.

(Continued on next page)
\end{abstract}

\footnotetext{
* Correspondence: liguilian@icdc.cn; guanchaxiang@csu.edu.cn

${ }^{2}$ State Key Laboratory for Infectious Disease Prevention and Control,

Collaborative Innovation Center for Diagnosis and Treatment of Infectious

Diseases, National Institute for Communicable Disease Control and

Prevention, Chinese Center for Disease Control and Prevention, Beijing

102206, People's Republic of China

'Department of Physiology, Xiangya School of Medicine, Central South

University, Changsha, Hunan 410078, China

Full list of author information is available at the end of the article
}

C C The Author(s). 2020 Open Access This article is licensed under a Creative Commons Attribution 4.0 International License, which permits use, sharing, adaptation, distribution and reproduction in any medium or format, as long as you give appropriate credit to the original author(s) and the source, provide a link to the Creative Commons licence, and indicate if changes were made. The images or other third party material in this article are included in the article's Creative Commons licence, unless indicated otherwise in a credit line to the material. If material is not included in the article's Creative Commons licence and your intended use is not permitted by statutory regulation or exceeds the permitted use, you will need to obtain permission directly from the copyright holder. To view a copy of this licence, visit http://creativecommons.org/licenses/by/4.0/ The Creative Commons Public Domain Dedication waiver (http://creativecommons.org/publicdomain/zero/1.0/) applies to the data made available in this article, unless otherwise stated in a credit line to the data. 


\begin{abstract}
(Continued from previous page)
Results: The results showed that the concordances between phenotypic DST and RDBH assay were 95\% for RIF, 92.8\% for $\mathrm{INH}, 84.7 \%$ for $\mathrm{SM}, 77.2 \%$ for EMB and the concordances between sequencing and RDBH assay were $97.8 \%$ for RIF, 98.8\% for INH, 99.1\% for SM, 93.4\% for EMB. Compared to the phenotypic DST results, the sensitivity and specificity of the RDBH assay for resistance detection were 92.4 and $98.5 \%$ for RIF, 90.3 and $97.3 \%$ for INH, 77.4 and $91.5 \%$ for SM, 61.4 and $85.7 \%$ for EMB, respectively; compared to sequencing, the sensitivity and specificity of the RDBH assay were 97.7 and $97.9 \%$ for RIF, 97.9 and $100.0 \%$ for INH, 97.8 and $100.0 \%$ for SM, 82.6 and $99.1 \%$ for EMB, respectively. The turnaround time of the RDBH assay was $7 \mathrm{~h}$ for testing 42 samples.
\end{abstract}

Conclusions: Our data suggested that the RDBH assay could serve as a rapid and efficient method for testing the resistance of $M$. tuberculosis against RIF, INH, SM and EMB, enabling early administration of appropriate treatment regimens to the affected drug resistant tuberculosis patients.

Keywords: Mycobacterium tuberculosis, Drug resistance, Reverse dot blot hybridization, Isoniazid, Rifampicin, Streptomycin, Ethambutol

\section{Background}

Mycobacterium tuberculosis infection remains a global public health threat due to its high risk of transmission, morbidity and mortality [1]. Timely case identification and appropriate treatment is of great significance in blocking the transmission of $M$. tuberculosis, especially for the prevention of multidrug-resistant (MDR) or extensively drug-resistant (XDR) M. tuberculosis emergence. Generally, culture-based phenotypic drug susceptibility testing (DST) methods are currently the gold standard for drug resistance detection and reliable and reproducible for certain anti-tuberculosis medicines, but these methods are time-consuming, e.g., MGIT medium based DST needs 4-13 days and LowensteinJensen (L-J) slants based DST needs 6 weeks [2]. Therefore, nucleic acid-based antibiotic susceptibility tests, which can be performed within 1 or 2 days, are increasingly considered as a diagnostic alternative.

Knowledge on the mutation profiles of drugs will be helpful to establish the molecular diagnosis assay for detecting the drugs resistance. Previous data shown that rifampicin (RIF) resistance is mainly attributed to the mutations within the RIF resistance-determining region (RRDR) of $r p o B$ (encoding $\beta$-subunit of RNA polymerase) with codons 531, 526 and 516 being the most prevalent sites [3-5]. Mutations in this region account for more than $90 \%$ of RIF resistance [5]. The molecular mechanisms of isoniazid (INH) resistance involve several genes in multiple biosynthetic networks and pathways. Mutation in the katG gene is the major cause for INH resistance, followed by inhA promoter and $o x y R$-ahpC intergenic region [6]. A study analyzed on $1219 \mathrm{INH}$ resistant isolates shown that the most frequent mutation loci were kat $G$ 315 (78.1\%), promoter inhA (-15) (22\%) [7]. Mutations in oxyR-ahpC intergenic region were found attributed to 10$15 \%$ INH resistance [7-9]. Mutations in rrs and rpsL genes, which are involved in the synthesis of 16S rRNA and the ribosomal protein S12, respectively, have been shown to be responsible for $50-95 \%$ streptomycin (SM) resistant strains $[4,7,8]$. The most frequent loci in rpsL and $r r s$ associated with SM resistance were $r p s L 43$, rpsL 88, rrs 513 loop and 912 loop $[4,7,8]$. Resistance to ethambutol (EMB) is mainly mediated by nucleotide changes in $e m b B$ particularly in codons 306 and 406 which accounted for $38-73 \%$ EMB resistance $[4,7,8]$. These resistanceassociated mutations provide the basis for molecular diagnostic approaches. In fact, the World Health Organization (WHO) recommends the use of molecular assays that target the specific mutations associated with resistance to certain anti-tuberculosis drugs [10, 11]. GenoType MTBDRplus (Hain Lifescience $\mathrm{GmbH}$, Nehren, Germany), which is a commercially available line probe assay, can detect $M$. tuberculosis complex as well as predict resistance to RIF and INH simultaneously from one to 16 samples within 5-6h, either in isolates or smear-positive specimens [11, 12]. Most recently, the FluoroType MTBDR (FluoroType) assay from Hain Lifescience $\mathrm{GmbH}$ is designed as a qualitative in vitro test for the automated detection of the M. tuberculosis complex and resistance to RIF and INH directly from sputum specimens [13]. Another new version (v2.0) of GenoType MTBDRsl assay (Hain Lifescience GmbH, Nehren, Germany) was developed to detect resistance to fluoroquinolones and second-line injectable drugs [14]. However, all of these assays did not include probes aimed for detecting mutations in $\operatorname{oxy} R-a h p C$, which has been reported accounted for $10-15 \%$ INH resistance [7-9]. Besides, detection of SM resistance is not included in GenoType methods. Therefore, it is necessary to establish a new assay to detect more multiple genes and mutations in a timely manner.

The in-house reverse dot blot hybridization (RDBH) assay has been widely used in the spoligotyping technique for M. tuberculosis lineage identification. Similarly, this technique had been successfully applied for the detection of mutations related to resistance to RIF and shown a sensitivity of $91.2 \%$ and a specificity of $98.3 \%$ comparing to phenotypic DST in our lab [15]. In order 
to further simplify consumables and make the procedure easier to perform, we improve the imaging method by replacing the enhanced chemiluminescence detection system described in previously report [15] with TMB (3, 3',5,5' -tetramethylbenzidine) reagent, which develop visible colorful spot directly. The RDBH method was further optimized to detect mutations conferring RIF, INH, EMB and SM resistance simultaneously by targeting in seven genes: $r p o B$ (for resistance to RIF), katG, inh $A$ promoter and $\operatorname{oxyR}$-ahpC (INH), rrs and rpsL (SM) and $e m b B$ (EMB), and evaluated its efficiency and accuracy to predict the resistance of four drugs by comparing to the phenotypic DST and sequencing results in 320 clinical M. tuberculosis isolates.

\section{Methods}

\section{Mycobacterium tuberculosis strains}

A total of $320 \mathrm{M}$. tuberculosis isolates were selected from the strain bank of the National Institute for Communicable Disease Control and Prevention, Chinese Center for Disease Control and Prevention. These isolates were obtained from 320 adult patients with pulmonary tuberculosis from 2005 to 2011 from institutes for tuberculosis control and prevention as well as tuberculosis hospitals distributed in six provincial-level administration divisions (PLADs) of China. The numbers isolated from each PLAD were with the following: Fujian, 76; Henan, 17; Hunan, 70; Inner Mongolia: 15; Sichuan, 34; Tibet, 108. H37Rv (ATCC 27294) was used as the reference strain.

All the strains were stored in physiological saline containing $50 \%$ glycerol at $-70^{\circ} \mathrm{C}$. Prior to characterizing the drug susceptibility, the strains were recovered on L-J medium for 4 weeks at $37^{\circ} \mathrm{C}$. The isolate profiles of drug susceptibility were reevaluated in our laboratory by the proportion method using L-J slants with the following: $0.2 \mu \mathrm{g} / \mathrm{ml}$ for INH, $40 \mu \mathrm{g} / \mathrm{ml}$ for RIF, $4 \mu \mathrm{g} / \mathrm{ml}$ for SM, and $2 \mu \mathrm{g} / \mathrm{ml}$ for EMB [16]. Of 320 isolates, 78 were susceptible to the four drugs, 23 were mono-INH resistant, eight were mono-SM resistant, 11 were mono-RIF resistant, 160 were MDR isolates (resistant to at least RIF and INH), 40 were poly-drug resistant (resistant to more than one drug but not MDR). In total, 206 were INH-resistant, 185 were RIF-resistant, 83 were EMB-resistant and 155 were SM-resistant.

\section{Genomic DNA extraction}

M. tuberculosis genomic DNA was extracted from fresh cultures growing on L-J slants. The bacterial cells were harvested and transferred to microcentrifuge tubes containing $200 \mu \mathrm{l}$ TE buffer $(10 \mathrm{mmol} / \mathrm{L}$ Tris- $\mathrm{HCl}$ and 1 $\mathrm{mmol} / \mathrm{L}$ EDTA, $\mathrm{pH} 8.0$ ), then inactivated in a $95^{\circ} \mathrm{C}$ water bath for $10 \mathrm{~min}$ and incubated at $85^{\circ} \mathrm{C}$ for $30 \mathrm{~min}$. After centrifugation for $5 \mathrm{~min}$ at $13523 \times g$, the supernatant containing DNA was collected and stored at $-20^{\circ} \mathrm{C}$ for further use.

\section{Multiplex PCRs}

The RDBH were designed based on multiplex PCRs. Seven PCR primer pairs (rpoB, katG, inhA promoter, oxyR-ahpC, rpsL, rrs and embB), biotinylated at the 5' end were designed to work together in a multiplex reaction. The primer sequences and amplicon sizes were listed in Table 1. Amplifications were performed in a final volume of $50 \mu \mathrm{l}$ containing $25 \mu \mathrm{l} 2 \times$ Hot Start Taq Master Mix (Sinobio, Shanghai, China), $2 \mu$ DMSO, 10$100 \mathrm{ng}$ of genomic DNA, forward and reverse primers with following concentraions (rpoB, $0.12 \mu \mathrm{mol} / \mathrm{L}$; katG, $0.18 \mu \mathrm{mol} / \mathrm{L}$; inh $A$ promoter, $0.2 \mu \mathrm{mol} / \mathrm{L} ;$ oxyR-ahpC, $0.2 \mu \mathrm{mol} / \mathrm{L} ; \quad r p s L, \quad 0.3 \mu \mathrm{mol} / \mathrm{L} ; \quad r r s, \quad 0.1 \mu \mathrm{mol} / \mathrm{L} ; \quad e m b B$, $0.24 \mu \mathrm{mol} / \mathrm{L})$. The cycling condition was as follows: 10 min at $94{ }^{\circ} \mathrm{C}, 35$ cycles of $1 \mathrm{~min}$ at $94{ }^{\circ} \mathrm{C}, 1 \mathrm{~min}$ at $63^{\circ} \mathrm{C}$ and $140 \mathrm{~s}$ at $72{ }^{\circ} \mathrm{C}$, and $10 \mathrm{~min}$ at $72{ }^{\circ} \mathrm{C}$. The PCR products were analyzed by electrophoresis using $3 \%$ agarose gels at $120 \mathrm{~V}$ for $80 \mathrm{~min}$.

\section{RDBH assay}

By targeting the major gene mutation sites conferring drug resistance to four anti-tuberculosis drugs including INH, RIF, SM and EMB, 15 probes detecting wild type (WT) sequences and 18 probes detecting mutant (MT) sequences were newly designed by using Primer Premier v.5.0 (Premier Biosoft International, Palo Alto, USA). The lengths of probes were adjusted to guarantee the difference of the melting temperatures of all probes within $6{ }^{\circ} \mathrm{C}$ so that they could be processed under the same hybridization and washing conditions. All the probes were covalently bonded to the negatively charged nylon membrane (Biodyne C, Pall Corporation, USA). $30 \mu$ of each PCR product was diluted in $140 \mu \mathrm{l} 5 \times$ saline-sodium phosphate-ethylenediaminetetraacetic acid (SSPE) $/ 0.1 \%$ sodium dodecyl sulfate (SDS) buffer, heat-denatured at $100^{\circ} \mathrm{C}$ for 10 min then immediately cooled on ice. The denatured single-stranded DNA was applied on the membrane in the miniblotter slots (Immunetics, Cambridge, MA, USA) and hybridized with probes at $60^{\circ} \mathrm{C}$ for $1 \mathrm{~h}$. The membrane was washed twice with $2 \times \operatorname{SSPE} / 0.5 \%$ SDS buffer at $50^{\circ} \mathrm{C}$ and subsequently incubated at $42{ }^{\circ} \mathrm{C}$ for $40 \mathrm{~min}$ with $20 \mathrm{ml} 2 \times \mathrm{SSPE} / 0.5 \%$ SDS containing 1:4000 diluted peroxidase (POD). Then the unbound conjugate was removed by washing twice in $2 \times \mathrm{SSPE} /$ $0.5 \%$ SDS for $10 \mathrm{~min}$ at $42^{\circ} \mathrm{C}$, and rinsed once with $2 \times$ SSPE for $5 \mathrm{~min}$ at room temperature. Finally the membrane was developed by incubating with $1 \mathrm{ml}$ TMB (Beyotime, Shanghai, China) reagent for $5 \mathrm{~min}$ in the dark.

A clear visible blue-green spot was recorded as positive. Clinical isolate was considered to be susceptible to the drug when the WT probes reacted positively while the MT probes were negative. When the mutant probe had a stronger color than the corresponding WT probe, the strain was considered to be a mutant genotype and 
Table 1 Primers designed for multiplex PCRs and sequencing

\begin{tabular}{|c|c|c|c|c|}
\hline Drug & Gene & Primer & Sequence $\left(5^{\prime} \rightarrow 3^{\prime}\right)$ & Amplicon size (bp) \\
\hline \multirow[t]{2}{*}{ RIF } & $r p o B$ & rpoB-F & GGTCGCCGCGATCAAGGAGT & 228 \\
\hline & & $r p o B-\mathrm{R}$ & GAGCCGATCAGACCGATGTT & \\
\hline \multirow[t]{6}{*}{$\mathrm{INH}$} & katG & katG-F & CAGATGGGCTTGGGCTGGAA & 152 \\
\hline & & katG-R & TTCGTCAGCTCCCACTCGTAGC & \\
\hline & $\operatorname{inh} A$ & $\operatorname{inh} A-\mathrm{F}$ & TGGTCGAAGTGTGCTGAGTC & 193 \\
\hline & & $\operatorname{inh} A-\mathrm{R}$ & TCCGGTAACCAGGACTGAAC & \\
\hline & oxyR-ahpC & oxyR-ahpC-F & GCAGTCACAACAAAGTCAGCTCTG & 401 \\
\hline & & oxyR-ahpC-R & ACAGGTCACCGCCGATGAGA & \\
\hline \multirow[t]{4}{*}{ SM } & $r p s L$ & $\operatorname{rps} L-\mathrm{F}$ & TTGTGGTTGCTCGTGCCTG & 635 \\
\hline & & $\operatorname{rps} L-F$ & CAACTGCGATCCGTAGACCG & \\
\hline & rrs & $r r s-\mathrm{R}$ & CTCTCGGATTGACGGTAGGTGG & 540 \\
\hline & & $r r s-\mathrm{F}$ & GCGTCCTGTGCATGTCAAACC & \\
\hline \multirow[t]{2}{*}{ EMB } & $e m b B$ & $e m b B-F$ & CGTGGTGATATTCGGCTTCCTG & 493 \\
\hline & & embB-R & CTGCACACCCAGTGTGAATGCG & \\
\hline
\end{tabular}

RIF Rifampicin, INH Isoniazid, SM Streptomycin, EMB Ethambutol

therefore resistant to the drug. A strain without positive spots of WT probes was recognized to have a specific mutation, and interpreted as resistant to the drug. Two $\mathrm{H} 37 \mathrm{Rv}$ pan-susceptible samples were used as positive controls and water was employed as negative control. The assay was performed and read in a double-blind way. For the disagreement results between two readers, a third reader was included to make the final decision. The results from the RDBH assay were compared to that obtained by proportion method (phenotypic DST) and sequencing.

\section{Standardization and validation}

H37Rv reference strain (ATCC 27294) and several M. tuberculosis clinical isolates with known mutations were used to select the optimal probe sequences and standardize the probe concentrations as well as assay conditions. Over a period of 2 years, series of experimental conditions according to the previous study of our lab, with varying probe concentrations $(0.1-1 \mu \mathrm{mol} / \mathrm{L})$, different hybridization temperatures $\left(50-65^{\circ} \mathrm{C}\right)$, washing temperatures $\left(45-60^{\circ} \mathrm{C}\right)$ and color-developing systems including alkaline phosphatase-nitroblue tetrazolium chloride/5bromo-4-chloro-3-indolyl pholsphate (AP-NBT/BCIP) and POD-TMB were performed in pilot experiments. A total of $320 \mathrm{M}$. tuberculosis clinical isolates was used to assess the performance of the RDBH assay, and the results were compared to that obtained by phenotypic DST and sequencing methods.

\section{Sequencing}

Mutations in seven genes or regions (rpoB, katG, inh $A$ promoter, oxyR-ahpC, rpsL, rrs 513 loop and embB) were also determined by sequencing. The primer sequences and concentrations used for separate PCR were equal to that used in multiplex-PCRs with the exception of primers of kat $G$ for obtaining longer DNA sequence: forward- AATCGATGGGCTTCAAGACG, reverseCTCGTAGCCGTACAGGATCTCG [17]. The PCR products of each gene were characterized by sequencing using the forward primers on an ABI Prism 3730 automated DNA sequencer (ABI Prism, Carlsbad, CA, USA). The resulting DNA sequences were analyzed using the basic local alignment search tool (http://www.ncbi.nih. gov/BLAST), and the specific mutations in protein sequences of the individual isolates were identified.

\section{Data analysis}

The Pearson's chi-square test was used to compute the statistical measures of sensitivity, specificity, positive predictive value (PPV), negative predictive value (NPV), and concordance of the RDBH assay using the phenotypic DST or sequencing as the gold standard method. The consistency analysis on the results of the different methods was conducted by Kappa identity test. The Kappa value was interpreted as follows: $<0.4$, limited; $0.41-0.75$, moderate; $\geq 0.75$, excellent [18]. All statistical analyses were performed using SPSS 18.0 software (SPSS Inc., Chicago, IL, USA).

\section{Results}

At the present study, we built a RDBH assay based on multiplex PCRs and evaluated its accuracy comparing with phenotypic DST and sequencing. The RDBH assay can test up to $42 M$. tuberculosis DNA samples at a time, the turnaround time from the beginning of multiplex PCRs to provide resistance results of isolates was $7 \mathrm{~h}$. The results of agarose gel electrophoresis shown that each target fragment was successfully amplified. A total of 320 M. tuberculosis were accessed by the RDBH assay. 
The $\mathrm{RDBH}$ results were determined according to the blot on the hybrid membrane, the interpretation on INH susceptibility between two readers was identical, whilst disagreements were found on five, six and six out of 320 isolates on SM, EMB and RIF susceptibility interpretations, respectively, and the third reader was needed to determin the final results. Figure 1 showed the RDBH membrane results of 41 isolates.

The performance of the RDBH assay compared with phenotypic DST and sequencing was summarized in Table 2 and Table 3, respectively. For detecting resistance to various drugs, the concordance between the RDBH assay and the phenotype DST varied from 77.2-95.0\%, with the Kappa values ranging from $0.43-0.90$, whlist the concordance between the RDBH assay and sequencing varied from 93.499.1\%, with the Kappa values ranging from 0.85-0.98.

\section{Comparisons of the RDBH assay, phenotypic DST and sequencing for rifampicin resistance detection}

Compared to the results of phenotypic DST, 171 (92.4\%) out of 185 phenotypic RIF resistant strains were identified as RIF-resistant isolates by the RDBH assay (Table 2).
Among 14 inconsistent isolates, 10 were not found mutations in $r p o B$ by sequencing. The remaining four isolates were identified as RIF-sensitive by the RDBH assay but shown harboring mutations in rpoB 513, 522 or 529 by sequencing. Of 135 phenotypic RIF-susceptible isolates, 133 and 2 were identified as RIF susceptible and resistant by the $\mathrm{RDBH}$ assay, respectively. The two inconsistent isolates were subsequently confirmed by sequencing to have mutations at codon 511 and 531 respectively.

Sequencing results showed that $174 \mathrm{M}$. tuberculosis strains were found to have alterations in the RRDR of rpoB. The most predominant mutations in $r p o B$ among these isolates were in the codons 531, 526 and 533, which were found in $82.2 \%$ $(152 / 185)$ phenotypic RIF-resistant isolates totally. Of the 174 M. tuberculosis carried mutations in rpoB, 170 (97.7\%) were identified as mutated and determined as RIF resistant by the RDBH assay (Table 3). Three out of 151 strains carried wild type $r p o B$ confirmed by sequencing were diagnosed as mutated strains and determined as RIF resistant by the RDBH assay, two of which were found negative results in the 509$514 \mathrm{WT}$ probe and identified as mutated strains, the remaining one showed mutation at codon 531 .

\section{2}

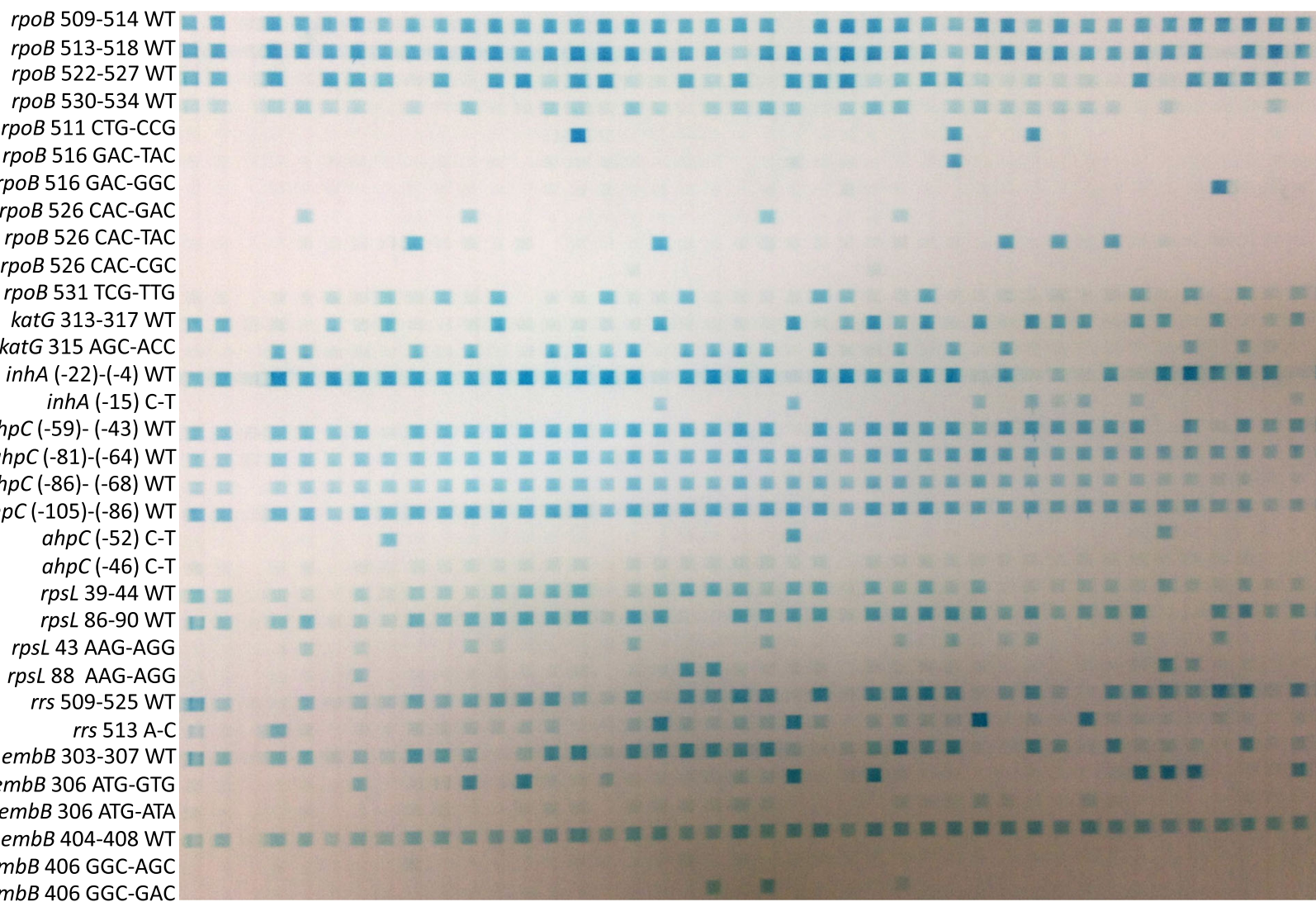
embB 406 GGC-GAC

Fig. 1 The hybridized image detected with the reverse dot blot hybridization assay. Note: lanes 1 to 2: H37Rv reference strain; lanes 3: Negative control; lanes 4 to 42: Mycobacterium tuberculosis clinical isolates. The detail information on how to interprete the results of the RDBH assay, the phenotypic resistance and sequencing results for $41 \mathrm{M}$. tuberculosis were shown in supplemental Table 1 
Table 2 The accuracy performance of RDBH assay compared to phenotypic DST in Mycobacterium tuberculosis clinical strains

\begin{tabular}{|c|c|c|c|c|c|c|c|c|c|}
\hline \multirow[t]{2}{*}{ Drug } & \multirow{2}{*}{$\begin{array}{l}\text { Phenotypic } \\
\text { DST }\end{array}$} & \multicolumn{8}{|c|}{ RDBH assay } \\
\hline & & $\mathrm{R}$ & $S$ & Sensitivity (\%) & Specificity (\%) & PPV (\%) & NPV (\%) & Concordance (\%) & Kappa value \\
\hline \multirow[t]{2}{*}{ RIF } & R & 171 & 14 & 92.4 & 98.5 & 98.8 & 90.5 & 95.0 & 0.90 \\
\hline & $S$ & 2 & 133 & & & & & & \\
\hline \multirow[t]{2}{*}{$\mathrm{INH}$} & R & 186 & 20 & 90.3 & 97.3 & 98.4 & 84.7 & 92.8 & 0.85 \\
\hline & S & 3 & 111 & & & & & & \\
\hline \multirow[t]{2}{*}{ SM } & R & 120 & 35 & 77.4 & 91.5 & 90.0 & 81.1 & 84.7 & 0.69 \\
\hline & S & 14 & 151 & & & & & & \\
\hline \multirow[t]{2}{*}{ EMB } & R & 51 & 32 & 61.4 & 82.7 & 55.4 & 86.0 & 77.2 & 0.43 \\
\hline & S & 41 & 196 & & & & & & \\
\hline
\end{tabular}

S Susceptible, $R$ Resistant, PPV Positive predictive value, NPV Negative predicte value, RIF Rifampicin, INH Isoniazid, SM Streptomycin, EMB Ethambutol, RDBH Reverse dot blot hybridization, DST Drug susceptibility testing

\section{Comparisons of the RDBH assay, phenotypic DST and sequencing for isoniazid resistance detection}

A total of 206 phenotypic INH-resistant and 114 INHsensitive clinical isolates have been examined by the $\mathrm{RDBH}$ assay. One hundred and eighty-six out of 206 (90.3\%) phenotypic INH resistant isolates were identified as INH resistant by the $\mathrm{RDBH}$ assay (Table 2). Among the 22 phenotypic INH resistant but the $\mathrm{RDBH}$ assay identified as INH susceptible isolates, 20 were not found mutations in the sequenced $k a t G$, inh $A$ promoter and oxyR-ahpC intergenic region, two were found carrying mutations either in katG 315 or inhA (-13) by sequencing. One hundred and eleven out of 114 phenotypic INH susceptible strains indicated positive results in all of the WT probes of $k a t G$, inhA promoter and oxyR$a h p C$ and identified as INH susceptible by the RDBH assay. For the remaining three phenotypic INH susceptible isolates that were identified as resistant by the $\mathrm{RDBH}$ assay, were found to harbor mutations inhA C(-

15) $\mathrm{T}$ or katG Ser315Thr by sequencing.

According to the sequencing results, $193 \mathrm{M}$. tuberculosis isolates carried mutations in katG, inhA promoter, or $\operatorname{oxy} R-a h p C$. Of the three regions sequenced, the katG gene showed the highest frequency of mutations, found in $74.3 \%(153 / 206)$ phenotypic INH resistant isolates. Mutations in the inhA promoter and $a h p C$-oxyR intergenic region were only found in 26 (12.6\%) and 14 (6.8\%) phenotypic INH resistant $M$. tuberculosis isolates, respectively. Among $193 M$. tuberculosis isolates that carried mutations in katG, inhA promoter, or oxyR-ahpC based on sequencing, 189 (97.9\%) were identified as mutated and INH resistant by the RDBH assay (Table 3). None of 127 isolates carried wild types of $k a t G$, inhA promoter and oxyR-ahpC confirmed by sequencing were diagnosed as mutated strains and then recognized as INH susceptible by the RDBH assay.

In the present study, probes for detecting mutations in the $\operatorname{xxy} R-a h p C$ region were included. The results showed that the sensitivity of the RDBH assay compared to the phenotypic DST were increased from 83.5 to $90.3 \%$, and increased from 90.7 to $97.9 \%$ compared to sequencing.

\section{Comparisons of the RDBH assay, phenotypic DST and sequencing for streptomycin resistance detection}

As shown in Table 2, among 155 phenotypic SM resistant strains, 120 were identified as SM resistant by the

Table 3 The accuracy performance of RDBH assay compared to sequencing in Mycobacterium tuberculosis clinical strains

\begin{tabular}{|c|c|c|c|c|c|c|c|c|c|}
\hline \multirow[t]{2}{*}{ Drug } & \multirow[t]{2}{*}{ Sequencing } & \multicolumn{8}{|c|}{ RDBH assay } \\
\hline & & $\bar{R}$ & $S$ & Sensitivity (\%) & Specificity (\%) & PPV (\%) & NPV (\%) & Concordance (\%) & Kappa value \\
\hline \multirow[t]{2}{*}{ RIF } & $M$ & 170 & 4 & 97.7 & 97.9 & 98.3 & 97.3 & 97.8 & 0.96 \\
\hline & W & 3 & 143 & & & & & & \\
\hline \multirow[t]{2}{*}{$\mathrm{INH}$} & M & 189 & 4 & 97.9 & 100.0 & 100.0 & 96.9 & 98.8 & 0.97 \\
\hline & W & 0 & 127 & & & & & & \\
\hline \multirow[t]{2}{*}{ SM } & M & 134 & 3 & 97.8 & 100.0 & 100.0 & 98.4 & 99.1 & 0.98 \\
\hline & W & 0 & 183 & & & & & & \\
\hline \multirow[t]{2}{*}{ EMB } & M & 90 & 19 & 82.6 & 99.1 & 97.8 & 91.7 & 93.4 & 0.85 \\
\hline & W & 2 & 209 & & & & & & \\
\hline
\end{tabular}


$\mathrm{RDBH}$ assay. Of the 35 remaining isolates which were identified as SM susceptible by RDBH, 32 were confirmed to carry wild type of rpsL and rrs and 3 were found to carry mutations at codon 43 or 88 in rpsL by sequencing. Among 165 phenotypic SM susceptible strains, 151 were identified as SM susceptible by the $\mathrm{RDBH}$ assay. The remaining 14 isolates were detected to have alteration in $r p s L$ or $r$ rs gene by sequencing.

Sequencing results showed that $137 \mathrm{M}$. tuberculosis strains carried mutations in rpsL or rrs. Mutations rpsL Lys43Arg and Lys88Arg were the most prevalent mutations, found in $67.1 \%(104 / 155)$ of the phenotypic SM resistant isolates. Among $137 \mathrm{M}$. tuberculosis strains that possessed substitutions in rpsL or rrs according to the sequencing results, 134 (97.8\%) were accurately determined as mutated and identified as SM resistant by the RDBH assay (Table 3). For the three inconsistent isolates, two isolates which both had mutation of rpsL 43 AAG-AGG and one had mutation of rpsL 86 CGG- CAG were considered as non-mutated by RDBH assay. Meanwhile, all of the 183 isolates that did not show any mutations based on sequencing were correctly diagnosed as non-mutated and identifies as SM susceptible by the RDBH assay.

\section{Comparisons of the RDBH assay, phenotypic DST and sequencing for ethambutol resistance detection}

Among 83 phenotypic EMB resistant strains, 51 were identified as EMB resistant and 32 were identified as EMB susceptible by the RDBH assay (Table 2). Among the 32 inconsistent strains, 10 were found mutations in $e m b B$ by sequencing: four carried embB 406 GGC-GAC (Gly-Asp), two carried 306 ATG-ATA (Met-Ile), two carried 334 TACCAC (Tyr-His), one carried 319 TAT-TCT (Tyr-Ser), one carried 354 GAC-AAC (Asp-Asn). Of 237 phenotypic EMB susceptible isolates, 196 were identified as EMB susceptible by the RDBH assay. The remaining 41 isolates identified as EMB resistant by the $\mathrm{RDBH}$ assay, 40 were found mutations in $e m b B, 1$ were found to have wild type of $e m b B$.

Sequencing results showed that $109 \mathrm{M}$. tuberculosis strains, including 60 phenotypic EMB resistant and 49 phenotypic EMB susceptible isolates carried mutations in $e m b B$. Among these 109 isolates, 90 were correctly identified as mutated by the RDBH assay (Table 3). The remaining 19 isolates which could not been identified as mutated by the RDBH assay carried mutations in $e m b B$ found by sequencing as follows: seven carried 406 GGCGAC (Gly-Asp), two carried 406 GGC-AGC (Gly-Ser), two carried 306 ATG-ATA (Met-Ile), one carried 306 ATG-GTG (Met-Val), two carried 334 TAC-CAC (TyrHis), two carried 328 GAT-TAT (Asp-Tyr), one carried 319 TAT-TGT (Tyr-Cys), one carried 319 TAT-TCT (Tyr-Ser). Among $211 M$. tuberculosis isolates found no mutations in the sequenced region of $e m b B$, two were identified as mutated by the RDBH assay.

\section{Prediction on multidrug resistance by the RDBH assay} A total of 160 phenotypic MDR isolates and 160 phenotypic non-MDR isolates were examined by the RDBH assay. One hundred and thirty-one out of 160 phenotypic MDR isolates were correctly identified as MDR by the RDBH assay. According to the sequening results, 141 isolates carried mutations both in RIF resistant associated gene rроB and INH resistant associated genes katG, inhA promoter or oxyR-ahpC interegenic, and were recognized as genotypic MDR. Among the 141 genotypic MDR isolates, 130 were correctly identified as MDR by the RDBH assay. None of phenotypic nonMDR and one genotypic non-MDR isolate was misclassified as MDR by the RDBH assay.

\section{Discussion}

The emergence and spread of MDR tuberculosis and XDR tuberculosis pose a serious impediment to global tuberculosis control. Early selection of appropriate treatment is vital to achieve good prognosis for the patients. Currently, molecular diagnostic methods, based upon the identification of specific gene mutations associated with drug resistance, are the most promising techniques for rapid detection of drug resistant $M$. tuberculosis clinical strains. Recently, some commercial assays, such as GenoType MTBDRplus, GenoType MTBDRsl, FluoroType MTBDR (Hain Lifescience GmbH, Nehren, Germany), GeneXpert MTB/RIF, Xpert MTB/RIF Ultra Assay (Cepheid Corp., USA), started to be used in many countries [11-14, 19-21]. These methods have sensitivities ranging from 85.56 to $100 \%$ and specificities spanning $78.26-100 \%$ for RIF resistance, and sensitivities from 61.6 to $100 \%$ and specificities spanning $66.7-100 \%$ for INH resistance by testing culture isolates of M. tuberculosis compared with a culture-based DST reference standard $(11,21,22)$. While GeneXpert MTB/RIF and Xpert MTB/RIF Ultra is limited to the detection of RIF resistance [22], GenoType MTBDRplus is able to identify both INH and RIF resistance [11]. In this study, a $\mathrm{RDBH}$ assay was established to simultaneously diagnose RIF, INH, SM and EMB resistance. It takes only $7 \mathrm{~h}$ for identification after extraction DNA from L-J cultures and allows the simultaneous analysis of 42 clinical DNA samples for four drug resistance detection. In the present study, we combined the susceptibility results of RIF and INH from the RDBH assay, and found that $82 \%$ phenotypic MDR isolates and 92\% genotypic MDR isolates been correctly identified. The ability of the RDBH assay for predicting multidrug resistance is directly affected by its ability for predicting INH and RIF resistance.

As for RIF, four WT probes and seven MT probes were designed to target mutations in RRDR, 92.4\% (171/ 185) phenotypic RIF-resistant strains and 97.7\% (170/ 174) strains carrying mutations in $r p o B$ were successfully determined by this analysis. We attributed the high 
sensitivity mainly to the wide coverage of diverse of mutation types. Compared with the principle of GenoType MTBDRplus, our RDBH assay excluded MT probes targeting codon 505-509 mutation because of their low frequencies of occurrence [12], but added four additional MT probes that were able to detect common mutations of Leu511Pro, Asp516Gly, Asp516Tyr, and His526Arg. Previous studies from different geographical locations have evaluated the use of RDBH assay in detecting RIF resistance and reported the sensitivity ranging from 85.6 to $100 \%[15,23,24]$. In our set of strains, codon 531,526 and 533 were the most frequently detected sites of $r p o B$ mutations associated with RIF resistance, accounting for $45.9,29.1$, and $8.6 \%$ respectively, which were contrasted to many other reports shown that the three most prevalent mutation loci in rpoB were 531, 526 and 516 [7, 25]. In addition, mutations were not observed in 14 RIF-resistant isolates, suggesting that there may be mutations outside the RRDR or elsewhere in the genome. Alternatively, the resistance might be due to the other underlying mechanisms, such as drug efflux pump or decreased permeability of the outer membrane [26]. Moreover, seven double-loci mutations were detected successfully by our assay, indicating that our method also had good sensitivity in detecting multiple loci mutations.

Our results showed that the use of RDBH assay could detect $73.3 \%(151 / 206)$ of phenotypic INH resistant isolates due to the presence of katG315 mutation. This could be further increased to $90.3 \%(186 / 206)$ if both the $i n h A$ promoter and oxyR-ahpC intergenic region mutations were involved. The sensitivity of this study was comparable to that reported from Pakistan (90.6\%) [27] but much higher than that from China (80.25\%) [28] which both used GenoType MTBDRplus. We speculated that the difference between this study and another study from China [28] may be attributed to that the mutation prevalence of oxyR-ahpC intergenic region were showed in 5-20\% INH resistant $M$. tuberculosis in China [5, 29, 30], however, GenoType MTBDRplus did not include probes targeted in oxyR-ahpC intergenic region. Previous studies showed that mutations in oxyR-ahpC intergenic region could compensate for loss of KatG/CP activity caused by mutations in katG [31] or were directly associated with low level INH resistance [32]. In our study, a 6.8\% (14/206) increase in the sensitivity was obtained by involving probes for the $\operatorname{axy} R$-ahpC. However, our method failed to detect four INH-resistant isolates, which harbored mutations in katG Ala312Glu, katG Trp191Gly, inhA G(-13) T and

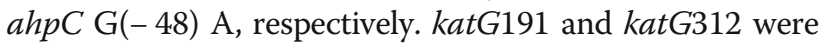
not included in the coverage of our targeted region due to the poor relevant to INH resistance, and isolates harbored mutations in inhA G(-13) T and ahpC G(-48) A showed ambiguous signals in the corresponding WT loci so that might be misjudged by readers of the RDBH assay. So, improvement of the specificity of the WT probes will be needed to avoid false negative results.

Farhat et al. reported that the $e m b B$ M306I and M306V mutations were significantly associated with INH resistance even after stratification by the EMB resistance status [7]. At the present study, we found that statistics significance were found between $e m b B$ mutations and INH resistance among the EMB susceptible isolates $\left(X^{2}=23.76, P=0.000\right)$ but not among EMB resistant isolates $\left(\chi^{2}=0.875, P=0.349\right)$. Since previous studies have showed that $e m b B$ M306I and M306V mutations were associated with INH resistance $[7,33]$, we added the results according to the probes targeted at $e m b B 306$ and found that the sensitivity increased from $90.3 \%(186 / 206)$ to $94.2 \%(194 / 206)$ whilst the specificity dropped from $97.3 \%(111 / 114)$ to $92.1 \%$ (105/114) compared to the phenotypic DST, so we suggested that embB306 mutation had limited effect on predicting INH resistance though there may be association between $e m b B$ mutations and INH resistance.

Regarding SM, RDBH assay was able to detect 120/155 SM phenotypic resistant samples (sensitivity $77.4 \%$ ) and 91.5\% susceptible isolates by detecting mutations in rpsL and rrs genes. There were 14 samples found to contain mutations by both the RDBH assay and sequencing but were susceptible to SM based on phenotypic DST results. Phenotypic DST assay (L-J based proportional method) was repeated for these samples to confirm the results. Similar phenomenon was also observed by Zhang et al. [34], which could be attributed to the low-level SM-resistance that showed falsenegative results by phenotypic DST. Regarding the 35 SM phenotypic resistant samples were detected as wild type by the $\mathrm{RDBH}$ assay, additional mechanisms which have been shown to be associated with SM resistance might be involved, e.g., cell membrane permeability changes [35], alterations in other genes, such as the gidB gene [36]. The mutations in rpsL were known to relate to high-level SM resistance whereas $r$ rs mutations were mainly related with lowlevel or intermediate-level SM resistance, identification of mutation types offered more information to understand the relationship between the phenotypic and genotypic feature of SM-resistant M. tuberculosis [37]. When compared with sequencing, the sensitivity and specificity of RDBH assay were $97.8 \%$ (134/137) and 100.0\% (183/183), respectively. Three isolates were diagnosed as unmutated by RDBH. Of these two harbored rpsL Lys43Arg mutation and the remaining one had rpsL Arg86Gln alteration. This suggests that further optimizing experimental conditions would improve the sensitivity.

As reported elsewhere, substitutions in codon embB306 are the predominant mechanism conferring EMB resistance, which accounted for $48.3-70.6 \%$ resistant isolates [38-41]. Among EMB-resistant isolates, we found that $72.3 \%(60 / 83)$ were observed to carry $e m b B$ 
mutation and 56.6\% (47/83) carried mutations in codon 306 of $e m b B$, which was consistent with the previous investigations [38-41]. So, we speculated that mutations in $e m b B$ was associated with EMB resistance. Using the RDBH assay, we obtained a moderate sensitivity (61.4\%) and specificity (85.7\%) compared with phenotypic DST and a higher sensitivity (82.6\%) and an excellent specificity (99.1\%) compared with sequencing. A multicenter evaluation by Mokrousov et al. [42] found lower sensitivity $(51.0 \%)$ and specificity $(82.6 \%)$ by only covering codon 306 . The improved detection efficiency might be attributed to that our assay covered three more probes targeted embB406. However, we also found that nine isolates carried mutation at embB406 were not identified by the RDBH assay, so the experimental conditions like reagent proportions, reaction temperatures should be further improved, or designing new probes targeted embB406 was needed. The discrepancy between the phenotypic DST and RDBH assay could be due to either a poor performance of the conventional DST method [43-45]. Clarifying the resistance mechanisms of EMB, such as $e m b A$, $e m b C$ and $e m b B$ codon 497, may also improve the performance of the molecular DST for predicting EMB resistance.

\section{Conclusions}

The multiplex PCRs based RDBH built in our study could determine three first-line drugs and one secondline drug resistance of 1 to $42 \mathrm{M}$. tuberculosis samples within $7 \mathrm{~h}$, and showed high consistency to phenotypic DST method and sequencing, suggesting that it is an outstanding diagnostic tool for simultaneously determining the resistance to RIF, INH, SM and EMB and is especially suitable for application in tuberculosis laboratories with heavy testing tasks.

\section{Supplementary information}

Supplementary information accompanies this paper at https://doi.org/10. 1186/s40249-020-00652-z.

Additional file 1: Supplemental Table 1 Interpretations of the RDBH assay, the phenotypic resistance and sequencing results for 41 Mycobacterium tuberculosis in Fig. 1.

\footnotetext{
Abbreviation

AP-NBT/BCIP: Alkaline phosphatase-nitroblue tetrazolium chloride/5-bromo4-chloro-3-indolyl pholsphate; DST: Drug susceptibility testing; EMB: Ethambutol; INH: Isoniazid; L-J: Lowenstein-Jensen; MDR: Multidrugresistant; MT: Mutant; NPV: Negative predictive value; PLAD: Provincial-level administration division; PPV: Positive predictive value; RDBH: Reverse dot blot hybridization; RIF: Rifampicin; RRDR: Rifampicin resistance-determining region; SDS: Sodium dodecyl sulfate; SM: Streptomycin; SSPE: Saline-sodium phosphate-ethylenediaminetetraacetic acid; TMB: 3, 3', 5, 5'Tetramethylbenzidine; WHO: World Health Organization; WT: Wild type; XDR: Extensively drug-resistant
}

\section{Acknowledgements}

The authors thank all staffs working in the province's tuberculosis hospitals or institutes for tuberculosis control of Fujian, Tibet, Henan, Sichuan, Hunan and Inner Mongolia provinces for supplying the clinical M. tuberculosis isolates.

\section{Authors' contributions}

This study was designed and conducted by GCX and LGL. WL and GQ wrote the first drafts of the manuscript. WL, GQ and WJH sequenced the isolates and performed the RDBH assay. LHC, LMC, JY, ZLL, ZXQ and LZG performed drug susceptibility testing. WKL collected $M$. tuberculosis strains and provided oversight for sequencing and bioinformatics support. GCX provided analysis support and key manuscript edits. LGL amended the manuscript. All authors commented on the manuscript draft and read and approved the final manuscript.

\section{Funding}

This study was supported by grants (Mega Projects of Research on the Prevention and Control of HIV/AIDS, Viral Hepatitis Infectious Diseases 2018ZX10103001-003012 and 2013ZX10003002-001) from the Ministry of Science and Technology, People's Republic of China, grant (2017zzts069) from Fundamental Research Funds for the Central Universities of Central South University, grant (81670014) from National Natural Science Foundation of China and grant (the project of Shanghai Public Health Clinical Center KY-GW-2017-10).

\section{Availability of data and materials}

The datasets used and/or analysed during the current study are available from the corresponding author on reasonable request.

\section{Ethics approval and consent to participate}

The study obtained approval from the Ethics Committee of National Institute for Communicable Disease Control and Prevention, Chinese Center for Disease Control and Prevention. The patients with tuberculosis included in the present research were given a Subject information sheet and they all gave written informed consent to participate in the study.

Consent for publication

Not applicable.

\section{Competing interests}

The authors declare that they have no competing interests.

\section{Author details}

${ }^{1}$ Department of Physiology, Xiangya School of Medicine, Central South University, Changsha, Hunan 410078, China. ${ }^{2}$ State Key Laboratory for Infectious Disease Prevention and Control, Collaborative Innovation Center for Diagnosis and Treatment of Infectious Diseases, National Institute for Communicable Disease Control and Prevention, Chinese Center for Disease Control and Prevention, Beijing 102206, People's Republic of China. ${ }^{3}$ Department of Molecular Biology, Shanghai Jiaotong University Affiliated Sixth People's Hospital, Shanghai 200233, People's Republic of China. ${ }^{4}$ Department of Clinical Laboratory, Shanghai Public Health Clinical Center, Fudan University, Shanghai 201508, China.

Received: 6 January 2020 Accepted: 24 March 2020

Published online: 16 April 2020

\section{References}

1. World Health Organization. Global tuberculosis report 2018. Geneva: World Health Organization; 2018

2. World Health Organization. Technical manual for drug susceptibility testing of medicines used in the treatment of tuberculosis. 2018. Licence: CC BYNC-SA 3.0 IGO.

3. Telenti A, Imboden P, Marchesi F, Lowrie D, Cole S, Colston MJ, et al. Detection of rifampicin-resistance mutations in Mycobacterium tuberculosis. Lancet. 1993;341(8846):647-50.

4. Li GL, Zhao DF, Xie T, Ju HF, Mu C, Zhao H, et al. Molecular characterization of drug-resistant Beijing family isolates of Mycobacterium tuberculosis from Tianjin, China. Biomed Environ Sci. 2010;23(3):188-93.

5. Zhao LL, Chen Y, Liu HC, Xia Q, Wu XC, Sun Q, et al. Molecular characterization of multidrug-resistant Mycobacterium tuberculosis isolates from China. Antimicrob Agents Chemother. 2014;58(4):1997-2005. 
6. Unissa AN, Subbian S, Hanna LE, Selvakumar N. Overview on mechanisms of isoniazid action and resistance in Mycobacterium tuberculosis. Infect Genet Evol. 2016:45:474-92.

7. Farhat MR, Sultana R, lartchouk O, Bozeman S, Galagan J, Sisk P, et al. Genetic determinants of drug resistance in Mycobacterium tuberculosis and their diagnostic value. Am J Respir Crit Care Med. 2016;194(5):621-30.

8. Miotto P, Tessema B, Tagliani E, Chindelevitch L, Starks AM, Emerson C, et al. A standardised method for interpreting the association between mutations and phenotypic drug resistance in Mycobacterium tuberculosis. Eur Respir J. 2017;50(6): 1701354.

9. Z Zhang H, Li D, Zhao L, Fleming J, Lin N, Wang T, et al. Genome sequencing of 161 Mycobacterium tuberculosis isolates from China identifies genes and intergenic regions associated with drug resistance. Nat Genet. 2013;45(10):1255-60.

10. World Health Organization. The use of molecular line probe assays for the detection of resistance to second-line anti-tuberculosis drugs, policy update. Geneva: World Health Organization; 2016. WHO/HTM/TB/2016.07.

11. World Health Organization. The use of molecular line probe assays for the detection of resistance to isoniazid and rifampicin, policy update. Geneva: World Health Organization. WHO/HTM/TB/2016.12.

12. Chen $C$, Kong W, Zhu L, Zhou Y, Peng H, Shao Y, et al. Evaluation of the GenoType((R)) MTBDRplus line probe assay on sputum-positive samples in routine settings in China. Int J Tuberc Lung Dis. 2014;18(9):1034-9.

13. de Vos M, Derendinger B, Dolby T, Simpson J, van Helden PD, Rice JE, et al. Diagnostic accuracy and utility of FluoroType MTBDR, a new molecular assay for multidrug-resistant tuberculosis. J Clin Microbiol. 2018;56(9). DOl: https://doi.org/10.1128/JCM.00531-18.

14. Tagliani E, Cabibbe AM, Miotto P, Borroni E, Toro JC, Mansjo M, et al. Diagnostic performance of the new version (v2.0) of GenoType MTBDRs assay for detection of resistance to fluoroquinolones and second-line injectable drugs: a multicenter study. J Clin Microbiol. 2015;53(9):2961-9.

15. Guo Q, Yu Y, Zhu YL, Zhao XQ, Liu ZG, Zhang YY, et al. Rapid detection of rifampin-resistant clinical isolates of Mycobacterium tuberculosis by reverse dot blot hybridization. Biomed Environ Sci. 2015;28(1):25-35.

16. World Health Organization. Updated Interim concentrations for first-line and second-line DST (as of May 2012). http://www.stoptb.org/wg/gli/assets/ documents/Updated\%20critical\%20concentration\%20table_1st\%20and\%202 nd\%20line\%20drugs.pdf (Accessed 17 Feb 2017).

17. Li Q, Ou XC, Pang Y, Xia H, Huang HR, Zhao B, et al. A novel automatic molecular test for detection of multidrug resistance tuberculosis in sputum specimen: a case control study. Tuberculosis (Edinb). 2017;105:9-12.

18. Landis JR, Koch GG. The measurement of observer agreement for categorical data. Biometrics. 1977;33(1):159-74.

19. Makhado NA, Matabane E, Faccin M, Pincon C, Jouet A, Boutachkourt F, et al. Outbreak of multidrug-resistant tuberculosis in South Africa undetected by WHO-endorsed commercial tests: an observational study. Lancet Infect Dis. 2018;18(12):1350-9.

20. Zar HJ, Workman $L$, Prins M, Bateman $L$, Mbhele SP, Whitman CB, et al. Tuberculosis diagnosis in children using Xpert ultra on different respiratory specimens. Am J Respir Crit Care Med. 2019;200(12):1531-8.

21. Boehme CC, Nabeta P, Hillemann D, Nicol MP, Shenai S, Krapp F, et al. Rapid molecular detection of tuberculosis and rifampin resistance. N Engl J Med. 2010;363(11):1005-15

22. Skenders GK, Holtz TH, Riekstina V, Leimane V. Implementation of the INNOLiPA Rif. TB(R) line-probe assay in rapid detection of multidrug-resistant tuberculosis in Latvia. Int J Tuberc Lung Dis. 2011;15(11):1546-52 i.

23. Senna SG, Gomes HM, Ribeiro MO, Kristki AL, Rossetti ML, Suffys PN. In house reverse line hybridization assay for rapid detection of susceptibility to rifampicin in isolates of Mycobacterium tuberculosis. J Microbiol Methods. 2006;67(2):385-9.

24. Morcillo N, Zumarraga M, Alito A, Dolmann A, Schouls L, Cataldi A, et al. A low cost, home-made, reverse-line blot hybridisation assay for rapid detection of rifampicin resistance in Mycobacterium tuberculosis. Int J Tuberc Lung Dis. 2002;6(11):959-65.

25. Shea J, Halse TA, Lapierre P, Shudt M, Kohlerschmidt D, Van Roey $P$, et al. Comprehensive whole-genome sequencing and reporting of drug resistance profiles on clinical cases of Mycobacterium tuberculosis in New York state. J Clin Microbiol. 2017:55(6):1871-82

26. Li G, Zhang J, Guo Q, Wei J, Jiang Y, Zhao X, et al. Study of efflux pump gene expression in rifampicin mono-resistant M.tuberculosis. J Antibiotics. 2015;68(7):431-5.
27. Siddiqui S, Brooks MB, Malik AA, Fuad J, Nazish A, Bano S, et al. Evaluation of GenoType MTBDRplus for the detection of drug-resistant Mycobacterium tuberculosis on isolates from Karachi. Pakistan PLoS One. 2019;14(8):e0221485.

28. Li Q, Dong HY, Pang Y, Xia H, Ou XC, Zhang ZY, et al. Multicenter evaluation of the molecular line probe assay for multidrug resistant Mycobacterium Tuberculosis detection in China. Biomed Environ Sci. 2015;28(6):464-7.

29. Luo D, Chen Q, Xiong G, Peng Y, Liu T, Chen X, et al. Prevalence and molecular characterization of multidrug-resistant M. tuberculosis in Jiangxi province, China. Sci Rep. 2019;9(1):7315

30. Liu L, Jiang F, Chen L, Zhao B, Dong J, Sun L, et al. The impact of combined gene mutations in inhA and $a h p C$ genes on high levels of isoniazid resistance amongst katG non-315 in multidrug-resistant tuberculosis isolates from China. Emerg Microbes Infect. 2018;7(1):183.

31. Sherman DR, Mdluli K, Hickey MJ, Arain TM, Morris SL, Barry CE 3rd, et al. Compensatory ahpC gene expression in isoniazid-resistant Mycobacterium tuberculosis. Science. 1996;272(5268):1641-3.

32. Zhang $Y$, Dhandayuthapani S, Deretic V. Molecular basis for the exquisite sensitivity of Mycobacterium tuberculosis to isoniazid. Proc Natl Acad Sci U S A. 1996;93(23):13212-6.

33. Sandgren A, Strong M, Muthukrishnan P, Weiner BK, Church GM, Murray MB Tuberculosis drug resistance mutation database. PLoS Med. 2009;6(2):e2.

34. Zhang T, Hu S, Li G, Li H, Liu X, Niu J, et al. Evaluation of the MeltPro TB/STR assay for rapid detection of streptomycin resistance in Mycobacterium tuberculosis. Tuberculosis (Edinb). 2015:95(2):162-9.

35. Nguyen L. Antibiotic resistance mechanisms in M. tuberculosis: an update. Arch Toxicol. 2016;90(7):1585-604.

36. Azadi D, Motallebirad T, Ghaffari K, Shojaei H. Mycobacteriosis and tuberculosis: laboratory diagnosis. Open Microbiol J. 2018;12:41-58.

37. Nhu NT, Lan NT, Phuong NT, Chau N, Farrar J, Caws M. Association of streptomycin resistance mutations with level of drug resistance and Mycobacterium tuberculosis genotypes. Int J Tuberc Lung Dis. 2012;16(4):527-31.

38. Zhao LL, Sun Q, Liu HC, Wu XC, Xiao TY, Zhao XQ, et al. Analysis of embCAB mutations associated with ethambutol resistance in multidrug-resistant mycobacterium tuberculosis isolates from China. Antimicrob Agents Chemother. 2015;59(4):2045-50.

39. Moure R, Espanol M, Tudo G, Vicente E, Coll P, Gonzalez-Martin J, et al. Characterization of the embB gene in Mycobacterium tuberculosis isolates from Barcelona and rapid detection of main mutations related to ethambutol resistance using a low-density DNA array. J Antimicrob Chemother. 2014;69(4):947-54.

40. Campbell PJ, Morlock GP, Sikes RD, Dalton TL, Metchock B, Starks AM, et al. Molecular detection of mutations associated with first- and second-line drug resistance compared with conventional drug susceptibility testing of Mycobacterium tuberculosis. Antimicrob Agents Chemother. 2011;55(5):2032-41.

41. Mokrousov I, Otten T, Vyshnevskiy B, Narvskaya O. Detection of embB306 mutations in ethambutol-susceptible clinical isolates of Mycobacterium tuberculosis from northwestern Russia: implications for genotypic resistance testing. J Clin Microbiol. 2002:40(10):3810-3.

42. Mokrousov I, Bhanu NV, Suffys PN, Kadival GV, Yap SF, Cho SN, et al. Multicenter evaluation of reverse line blot assay for detection of drug resistance in Mycobacterium tuberculosis clinical isolates. J Microbiol Methods. 2004;57(3):323-35.

43. Walker TM, Kohl TA, Omar SV, Hedge J, Del Ojo EC, Bradley P, et al. Wholegenome sequencing for prediction of Mycobacterium tuberculosis drug susceptibility and resistance: a retrospective cohort study. Lancet Infect Dis. 2015;15(10):1193-202.

44. Schon T, Miotto P, Koser CU, Viveiros M, Bottger E, Cambau E. Mycobacterium tuberculosis drug-resistance testing: challenges, recent developments and perspectives. Clin Microbiol Infect. 2017;23(3):154-60.

45. Zhang N, J.H. H, Zhao XQ, wan KL. Preliminary study on the appropriate concentrations of drug used in the drug-susceptibility test to detect the Ethambutol-resistant isolates of Mycobacterium tuberculosis. Chin J Zoonoses 2009;25(11):1049-1053 